Doug Geisler, Eva K. Grebel, and Dante Minniti, eds.

\title{
A Survey of M31 Globular Clusters using WFPC2 on board HST
}

\author{
R.M. Rich \\ Dept. of Phys. \& Astron., UCLA, Los Angeles, CA 90095-1562, USA \\ C.E. Corsi \\ Osservatorio Astronomico, V.le Parco Mellini 84, 00136 Roma, Italy \\ M. Bellazzini, L. Federici, C. Cacciari \\ Osservatorio Astronomico, Via Ranzani 1, 40127 Bologna, Italy \\ F. Fusi Pecci \\ Osservatorio Astronomico, Poggio dei Pini, 09012 Capoterra, Italy
}

\begin{abstract}
We report new, as yet unpublished HST/WFPC2 V, I photometry of 9 globular clusters in M31. These are part of a total sample of 19 clusters on M31 with well observed CMDs from HST. The clusters have the full range of horizontal branch morphologies from blue HB to red clump, but none are found with an extreme (blue only) horizontal branch. Plots of HB type vs $[\mathrm{Fe} / \mathrm{H}]$ are similar to those of Galactic clusters, including a hint of second parameter clusters. Sixteen halo fields adjacent to the observed clusters included in our images have been analysed. The M31 halo metallicity distribution peaks at $[\mathrm{Fe} / \mathrm{H}]=-0.7 \mathrm{dex}$ with a tail toward low metallicity, resembling the halo of NGC 5128; metal-rich giants appear to be centrally concentrated.
\end{abstract}

\section{The globular clusters}

At present, 22 globular clusters (GCs) have been observed with FOC or WFPC2 on board HST. We report here on the V (F555W) and I (F814W) yet unpublished photometry obtained by Rich and collaborators on the 9 GCs most recently observed (GO6671, PI: R.M. Rich). The aim of the project is to compare the stellar populations of the M31 and Milky Way cluster systems, and to measure $M_{V}^{H B}$ vs $[\mathrm{Fe} / \mathrm{H}]$ for a sample of clusters at the same distance. The data was processed using the HST pipeline, and reduced using the ROMAFOT package which is optimized for accurate photometry in crowded fields.

With the exclusion of 3 clusters from the total sample of 22 , because they are concentrated in the innermost region of the galaxy and their photometry does not allow the definition of good CMDs, there are now 19 CMDs of sufficiently good quality to afford some general analysis of the characteristics of the GC system in M31: 


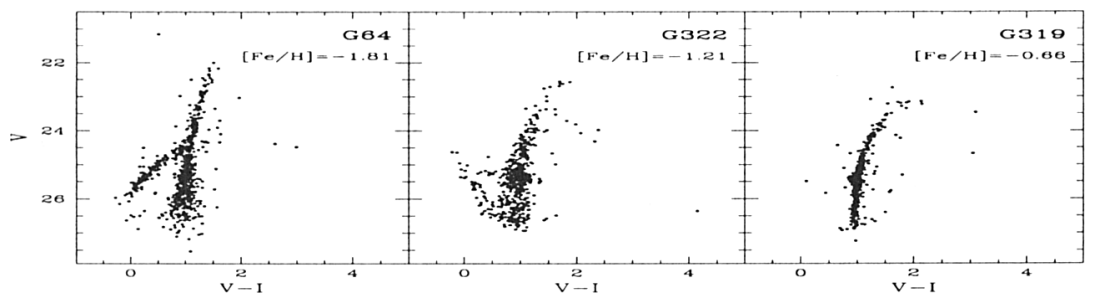

Figure 1. Romafot Photometry of 3 GCs in M31 spanning the full range in metallicity. The RGB slope and HB follow the usual Milky Way trends; G322 may be a second parameter cluster. The mean accuracy of the data is $\approx 0.02 \mathrm{mag}$ in $(\mathrm{V}, \mathrm{I}), \approx 0.03 \mathrm{mag}$ in $(\mathrm{V}-\mathrm{I})$ for $\mathrm{V}<24, \approx 0.06 \mathrm{mag}$ in $(\mathrm{V}, \mathrm{I})$, and $\approx 0.08 \mathrm{mag}$ in $(\mathrm{V}-\mathrm{I})$ for $\mathrm{V}>24$.

- Our clusters range over $-1.9<[\mathrm{Fe} / \mathrm{H}]<-0.6$ dex with metallicities from ground-based integrated spectra (Barmby et al. 2000). The red giant branch (RGB) morphology and color of the M31 clusters correlate with metallicity in the same way as for the Milky Way clusters.

- Horizontal branch (HB) morphologies range from a stubby red clump in the most metal-rich clusters to extended blue HBs at the metal poor end. We do not find any clusters with extreme blue HBs (such as M92). A plot of HB type as a function of $[\mathrm{Fe} / \mathrm{H}]$ is similar to that for the Galactic GCs, and hints at the existence of second parameter clusters.

- The dependence of HB luminosity on metallicity is found to be $M_{V}^{H B}=$ $0.22[\mathrm{Fe} / \mathrm{H}]+$ const where the zero point depends on the assumed M31 distance. We find a steeper slope (0.22) than the value of 0.13 found by Fusi Pecci et al. (1996) based on HST photometry of 8 M31 GCs.

We conclude that the M31 GCs show all signs of being very similar to the Milky Way GCs, with no significant anomalies. There is no strong indication of intermediate age GCs analogous to those found e.g. in the SMC.

\section{Photometry of the M31 halo field population}

We have photometry for sixteen fields in the M31 halo, thanks to the clusters falling on the PC only, making the 3 chips of the WFC available to image the field population. These have been reduced in the same way as the clusters, and a subsample of four of our CMDs is given in Figure 2, in order of increasing projected galactocentric distance. The descending red giant branches, indicative of high metallicity, persist even at $20 \mathrm{kpc}$ from the nucleus. 


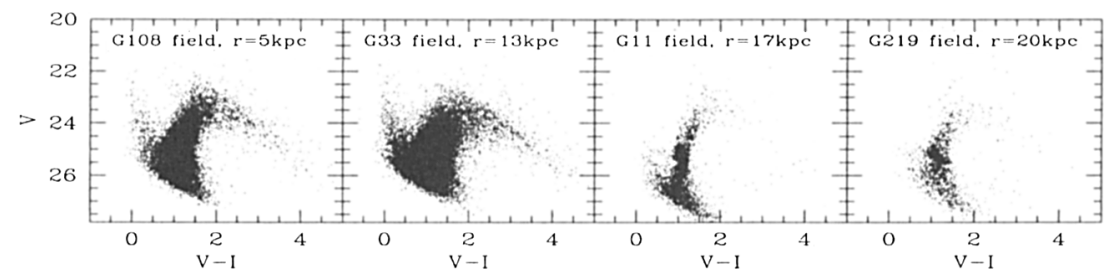

Figure 2. Color-Magnitude diagrams of 4 fields adjacent to GCs in M31. Blue sequences are disk stars in M31, while the descending red giant branch is indicative of high metallicity. The CMD more closely resembles the Milky Way bulge than it does a halo field, even at 20 $\mathrm{kpc}$ from the nucleus (G219 field).

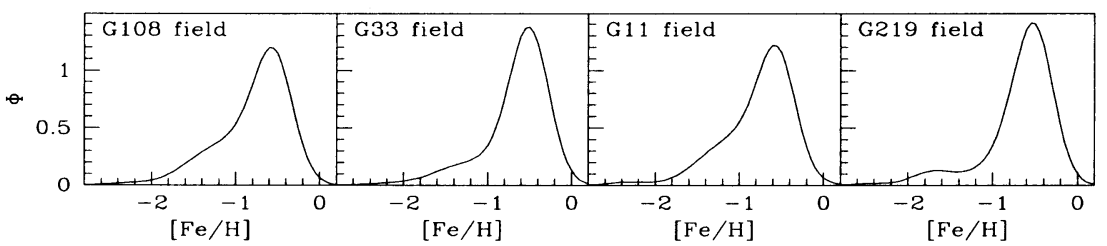

Figure 3. Generalized metallicity histograms for giants in the 4 halo fields shown in Fig.2; see text.

We estimate metallicities of the field giants by interpolating their colors between template GC giant branches of known metallicity. We see a strong metal rich peak at -0.6 in the metallicity distribution, with a tail reaching near -2 (Fig. 3). Varying the RGB templates and reddening does not change the fundamental form of the abundance distribution, which hints at a second mode near -1.3 . We find no evidence for a gradient in the mean or modal metallicity, but the fraction of metal rich/metal poor stars increases toward the nucleus. Our M31 halo metallicity is higher than that of the Milky Way halo, but the shape and mean agree with those in other galaxies, e.g. NGC5128 (Harris \& Harris, 2000). It is difficult to imagine how the accretion of low mass, low metallicity satellites (such as envisioned by CDM models) could produce such a metal rich halo population. Such high metallicities (even $20 \mathrm{kpc}$ from the nucleus) could be produced by a metal-enriched wind connected with the formation of the spheroid, with some fraction of halo material coming from dissolved satellites.

Support was provided by NASA through grant number GO-6671 from the STScI, which is operated by AURA, Inc., under NASA contract NAS5-26555.

\section{References}

Barmby, P., Huchra, J.P., Brodie, J.P., et al. 2000, AJ, 119, 727

Fusi Pecci, F., Buonanno, R., Cacciari, C., et al. 1996, AJ, 112, 1461

Harris, G.L.H., \& Harris, W.E. 2000, AJ, 120, 2423 